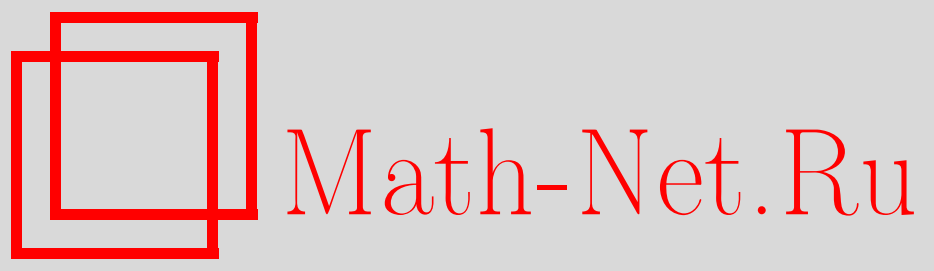

Ф. Али, Т. Ферозе, Пространство-время типа гравитационной волны с цилиндрической симметрией, ТМФ, 2017, том 193, номер 2, 343-355

DOI: https://doi.org/10.4213/tmf9148

Использование Общероссийского математического портала Math-Net.Ru подразумевает, что вы прочитали и согласны с пользовательским соглашением http://www.mathnet.ru/rus/agreement

Параметры загрузки:

IP: 18.234 .156 .22

26 апреля 2023 г., 13:11:12

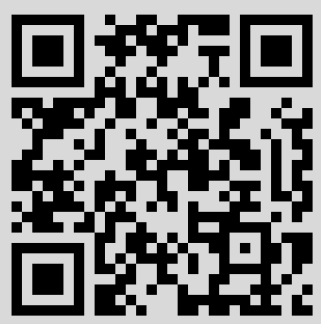




\title{
ПРОСТРАНСТВО-ВРЕМЯ ТИПА ГРАВИТАЦИОННОЙ ВОЛНЫ С ЦИЛИНДРИЧЕСКОЙ СИММЕТРИЕЙ
}

\begin{abstract}
Представлены симметрии Нётер для лагранжиана, задающего геодезические, в пространстве-времени с цилиндрической симметрией в конформном времени. Временной конформный фактор вводится в общее цилиндрически-симметричное пространство-время, чтобы сделать его нестатическим и затем найти приближенные симметрии Нётер действия, соответствующего лагранжиану. В первом приближении теории возмущений найдены все лагранжианы для цилиндрически-симметричных пространств-времен, для которых существуют приближенные симметрии Нётер.
\end{abstract}

Ключевые слова: симметрии Нётер, теорема Нётер, первые интегралы, полевые уравнения Эйнштейна, гравитационная волна.

DOI: https://doi.org/10.4213/tmf9148

\section{1. ВВЕДЕНИЕ}

Гравитационные волны являются распространяющимися флуктуациями гравитационных полей, т. е. возмущениями в пространстве-времени, порождаемыми в основном движениями массивных тел. Эти возмущения пространства-времени распространяются со скоростью света [1]-[5]. Каждое тело на пути такой волны ощущает воздействие приливно-отливной гравитационной силы, которая действует в направлении, перпендикулярном направлению распространения волн; эти силы изменяют расстояние между точками пространства-времени, и величина этих изменений пропорциональна расстоянию между точками. Гравитационные волны могут быть обнаружены приборами, которые измеряют индуцированные изменения длины. Частоты и амплитуды волн связаны с движением рассматриваемых масс. Таким образом, анализ гравитационных волн позволяет изучать источники, создающие эти волны. Эти волны являются полезным инструментом получения информации об источниках, которые производят эти волны.

*Department of Mathematics, Kohat University of Science and Technology, Kohat, Pakistan. E-mail: farhadmardan@gmail.com

${ }^{\dagger}$ School of Natural Sciences, National University of Sciences and Technology, Islamabad, Pakistan 
Энергия и импульс являются важными величинами, определение которых было фокусом многих исследований в области общей теории относительности. К сожалению, до сих пор нет общепринятого определения энергии и импульса в общей теории относительности, и эти величины, вообще говоря, не сохраняются [6]-[8]. Были сделаны различные попытки определить эти величины. Эйнштейн и Розен [9] показали, что гравитационные волны забирают энергию из источника; возникает основной вопрос, как определить эту энергию, которая передается гравитационной волной от источника. Симметрии Нётер играют важную роль в этом отношении [10], [11], так как существует однозначное соответствие между этими симметриями и законами сохранения [7], [12]. Симметрии Нётер используются для определения законов сохранения в цилиндрически-симметричном статическом пространстве-времени, в некоторых классах пространства-времени типа рр-волн, в пространстве-времени типа Годеля и для $f(R)$-гравитации [13]-[16]. Они также используются в расширенной гравитации и квантовой космологии [17], [18].

В последнее время приближенные симметрии Нётер использовались для определения приближенных законов сохранения в хаотичной гамильтоновой системе и в динамической системе [19]-[23]. Мы применили метод приближенных симметрий Нётер для нахождения цилиндрически-симметричных нестатических пространств-времен, которые не являются в точности пространствами-временами гравитационных волн, но являются приближенно пространствами-временами типа гравитационных волн, для которых $R_{\mu \nu} \rightarrow 0$ при $x \rightarrow \infty$. Пространства-времена Леви-Чивиты - цилиндрически-симметричные пространства-времена, которые являются плоскими пространствами-временами Риччи, но статическими [24] (не зависящими от времени), однако пространство-время гравитационной волны должно быть нестатическим. В настоящей работе мы рассматриваем цилиндрически-симметричное пространство-время, которое в результате воздействия со стороны временно́го конформного фактора становится нестатическим пространством-временем, т. е. оно не соответствует пространству-времени типа гравитационных волн. Это пространство-время рассматривается как приближенное пространство-время гравитационных волн, что дает нам информацию о поведении точного пространства-времени типа гравитационных волн.

Приближенные симметрии Нётер для лагранжиана общего цилиндрически-симметричного пространства-времени получены в первом приближении теории возмущений для изучения энергии и импульса. Мы рассматриваем цилиндрически-симметричные статические пространства-времена, так как они играют важную роль в изучении гравитационных волн [25], статическая часть которых обеспечивает сохранение энергии. Так как волны не могут быть статическими, мы вводим общий зависящий от времени конформный фактор $e^{\epsilon f(t)}$, где $\epsilon$ - малый произвольный параметр, а $f(t)$ - обобщенная функция $t$, для того чтобы сделать пространство-время нестатическим. С помощью разложения Тейлора получаем приближенную (возмущенную) в первом порядке цилиндрически-симметричную метрику и соответствующий приближенный лагранжиан. Этот приближенный лагранжиан затем используется для нахождения приближенных симметрий Нётер. Мы исследовали много случаев, в которых точные симметрии для статических пространств-времен появляются вместе с приближенными частями. Среди них времениподобный бесконечно 
малый производящий оператор $\partial / \partial t$ является основным фокусом нашей работы, который соответствует энергии материи в соответствующих пространствах-временах. Мы также находим некоторые другие приближенные симметрии Нётер, соответствующие другим законам сохранения, которые пропадают при формировании гравитационной волны.

Черные дыры являются одним из источников гравитационных волн. Возмущение, которое мы используем в этой статье, может быть применено в теории черных дыр. Мы исследовали этот тип возмущения в случае черной дыры Шварцшильда [26] и собираемся иссследовать этот тип возмущения в случае обобщенных цилиндрически-симметричных пространств-времен, так как они связаны с теорией черных дыр. В настоящей работе мы находим все те цилиндрически-симметричные пространства-времена, которые допускают конформный фактор, зависящий от времени. Мы надеемся, что эта работа будет полезна для понимания теории черных дыр. Наша будущая цель заключается в том, чтобы найти конформные при всех временах сферически-симметричные пространства-времена, которые непосредственно связаны с теорией черных дыр, так как черная дыра считается сферически-симметричной.

\section{2. ЦИЛИНДРИЧЕСКИ-СИММЕТРИЧНОЕ СТАТИЧЕСКОЕ ПРОСТРАНСТВО-ВРЕМЯ, ЕГО ЛАГРАНЖИАН И ВОЗМУЩЕНИЕ ПЕРВОГО ПОРЯДКА}

Обобщенное цилиндрически-симметричное статическое пространство-время и соответствующий лагранжиан даются следующими выражениями [27]:

$$
\begin{aligned}
d s_{\mathrm{e}}^{2} & =e^{\nu(r)} d t^{2}-d r^{2}-e^{\mu(r)} d \theta^{2}-e^{\lambda(r)} d z^{2} \\
\mathcal{L}_{\mathrm{e}} & =e^{\nu(r)} \dot{t}^{2}-\dot{r}^{2}-e^{\mu(r)} \dot{\theta}^{2}-e^{\lambda(r)} \dot{z}^{2}
\end{aligned}
$$

Теперь, вводя обобщенный зависящий от времени конформный фактор $e^{\epsilon f(t)}$, мы имеем

$$
d s^{2}=e^{\epsilon f(t)} d s_{\mathrm{e}}^{2}, \quad \mathcal{L}=e^{\epsilon f(t)} \mathcal{L}_{\mathrm{e}}
$$

В первом приближении возмущенная метрика и лагранжиан в развернутой форме записываются как

$$
\begin{aligned}
d s^{2}= & e^{\nu(r)} d t^{2}-d r^{2}-e^{\mu(r)} d \theta^{2}-e^{\lambda(r)} d z^{2}+ \\
& \quad+\epsilon f(t)\left(e^{\nu(r)} d t^{2}-d r^{2}-e^{\mu(r)} d \theta^{2}-e^{\lambda(r)} d z^{2}\right), \\
\mathcal{L}= & e^{\nu(r)} \dot{t}^{2}-\dot{r}^{2}-e^{\mu(r)} \dot{\theta}^{2}-e^{\lambda(r)} \dot{z}^{2}+\epsilon f(t)\left(e^{\nu(r)} \dot{t}^{2}-\dot{r}^{2}-e^{\mu(r)} \dot{\theta}^{2}-e^{\lambda} \dot{z}^{2}\right)= \\
= & \mathcal{L}_{\mathrm{e}}+\epsilon \mathcal{L}_{\mathrm{a}},
\end{aligned}
$$

где $\mathcal{L}_{\mathrm{e}}$ и $\mathcal{L}_{\mathrm{a}}$ - точная и возмущенная части лагранжиана соответственно. Эта процедура дает приближенные части в симметриях Нётер, которые будут соответствовать приближенным законам сохранения в соответствующих пространствах-временах. 


\section{3. ПРИБЛИЖЕННАЯ СИММЕТРИЯ НЁТЕР}

Оператор $\mathbf{X}$ называется производящим оператором приближенных симметрий Нётер, если он удовлетворяет условию [7]

$$
\mathbf{X}^{1} \mathcal{L}+(D \xi) \mathcal{L}=D A
$$

где $\mathbf{X}^{1}$ является продолжением первого порядка первого же порядка приближенной симметрии Нётер $\mathbf{X}=\mathbf{X}_{\mathrm{e}}+\epsilon \mathbf{X}_{\mathrm{a}}$,

$$
\begin{aligned}
& \mathbf{X}_{\mathrm{e}}=\xi_{\mathrm{e}} \frac{\partial}{\partial s}+\eta_{\mathrm{e}}^{0} \frac{\partial}{\partial t}+\eta_{\mathrm{e}}^{1} \frac{\partial}{\partial r}+\eta_{\mathrm{e}}^{2} \frac{\partial}{\partial \theta}+\eta_{\mathrm{e}}^{3} \frac{\partial}{\partial z} \\
& \mathbf{X}_{\mathrm{a}}=\xi_{\mathrm{a}} \frac{\partial}{\partial s}+\eta_{\mathrm{a}}^{0} \frac{\partial}{\partial t}+\eta_{\mathrm{a}}^{1} \frac{\partial}{\partial r}+\eta_{\mathrm{a}}^{2} \frac{\partial}{\partial \theta}+\eta_{\mathrm{a}}^{3} \frac{\partial}{\partial z}
\end{aligned}
$$

являются точной и приближенной частями $\mathbf{X}$ соответственно, а $D$ - дифференциальный оператор вида

$$
D=\frac{\partial}{\partial s}+\dot{t} \frac{\partial}{\partial t}+\dot{r} \frac{\partial}{\partial x}+\dot{\theta} \frac{\partial}{\partial \theta}+\dot{z} \frac{\partial}{\partial z},
$$

$A=A_{\mathrm{e}}+\epsilon A_{\mathrm{a}}$, где $A_{\mathrm{e}}$ и $A_{\mathrm{a}}$ - точная и приближенная части калибровочной функции $A$. Уравнение (3) разбивается на две части следующим образом:

$$
\begin{aligned}
\mathbf{X}_{\mathrm{e}} \mathcal{L}_{\mathrm{e}}+\left(D \xi_{\mathrm{e}}\right) \mathcal{L}_{\mathrm{e}} & =D A_{\mathrm{e}} \\
\mathbf{X}_{\mathrm{a}}^{1} \mathcal{L}_{\mathrm{e}}+\mathbf{X}_{\mathrm{e}}^{1} \mathcal{L}_{\mathrm{a}}+\left(D \xi_{\mathrm{e}}\right) \mathcal{L}_{\mathrm{a}}+\left(D \xi_{\mathrm{a}}\right) \mathcal{L}_{\mathrm{e}} & =D A_{\mathrm{a}}
\end{aligned}
$$

Здесь, $\eta_{\mathrm{e}}^{i}, \eta_{\mathrm{a}}^{i}, \xi_{\mathrm{e}}, \xi_{\mathrm{a}}, A_{\mathrm{e}}$ и $A_{\mathrm{a}}$ являются функциями $s, t, r, \theta, z$, a $\dot{\eta}_{\mathrm{e}}^{i}, \dot{\eta}_{\mathrm{a}}^{i}-$ функциями $s, t, r, \theta, z, \dot{t}, \dot{r}, \dot{\theta}, \dot{z}$, и точка над буквой означает дифференцирование по $s$. В настоящей работе мы ищем решения уравнения (7), так как эти решения дают все цилиндрически-симметричные пространства-времена, лагранжианы которых допускают приближенные симметрии Нётер. Из уравнения (7) мы получаем систему 19 симметрий Нётер, определяющих дифференциальные уравнения в частных производных, решения которых обеспечивают необходимые результаты.

\section{4. СИСТЕМА СИММЕТРИЙ НЁТЕР, УПРАВЛЯЮЩАЯ ДИФФЕРЕНЦИАЛЬНЫМИ УРАВНЕНИЯМИ В ЧАСТНЫХ ПРОИЗВОДНЫХ}

Из уравнения (7) мы получаем систему 19 дифференциальных уравнений в частных производных, которая приводится в приложении. Мы решаем эту систему с помощью точных решений цилиндрически-симметричного статического пространства-времени [28]. В настоящем разделе мы перечислим все те классы, для которых существуют приближенные симметрии Нётер.

4.1. Приближенные симметрии Нётер из лагранжиана цилиндрически-симметричного пространства-времени. Пространство-время вида

$$
d s^{2}=e^{\nu(r)} d t^{2}-d r^{2}-e^{\mu(r)} d \theta^{2}-e^{\lambda(r)} d z^{2}+\frac{\epsilon t}{\alpha}\left(e^{\nu(r)} d t^{2}-d r^{2}-e^{\mu(r)} d \theta^{2}-e^{\lambda(r)} d z^{2}\right)
$$


ТАБЛИЦА 1

\begin{tabular}{|c|c|}
\hline Симметрии & Первые интегралы \\
\hline $\mathbf{X}_{1}$ & $E=-2 e^{\nu(x)} \dot{t}-\frac{\epsilon}{\alpha}\left(2 t \dot{t} e^{\nu(x)}-\widetilde{\mathcal{L}} s\right)$ \\
\hline $\mathbf{X}_{2}$ & $P_{\theta}=2\left(1+\frac{\epsilon t}{\alpha}\right) e^{\mu(r)} \dot{\theta}$ \\
\hline $\mathbf{X}_{3}$ & $P_{z}=2\left(1+\frac{\epsilon t}{\alpha}\right) e^{\lambda(r)} \dot{z}$ \\
\hline $\mathbf{X}_{4}$ & $\widetilde{\mathcal{L}}=\left(1+\frac{\epsilon t}{\alpha}\right)\left(e^{\nu(x)} \dot{t}^{2}-\dot{r}^{2}-e^{\mu(x)} \dot{\theta}^{2}-e^{\lambda(r)} \dot{z}^{2}\right)$ \\
\hline
\end{tabular}

допускает только четыре симметрии Нётер для функций $\nu(r), \mu(r)$ и $\lambda(r)$ общего вида, которые записываются как

$$
\mathbf{X}_{1}=\frac{\partial}{\partial t}+\frac{\epsilon s}{\alpha} \frac{\partial}{\partial s}, \quad \mathbf{X}_{2}=\frac{\partial}{\partial \theta}, \quad \mathbf{X}_{3}=\frac{\partial}{\partial z}, \quad \mathbf{X}_{4}=\frac{\partial}{\partial s}
$$

Первые интегралы, соответствующие этим симметриям, приведены в табл. 1. Интегралы $P_{\theta}, P_{z}$ и $\widetilde{\mathcal{L}}$, приведенные в табл. 1 , остаются одинаковыми во всех классах, приведенных в этой статье; изменяются только функции $\nu(r), \mu(r)$ и $\lambda(r)$.

Мы имеем следующие классы для шести симметрий Нётер.

1. Первый класс шести симметрий Нётер:

$$
\begin{aligned}
d s^{2}=\left(\frac{r}{\alpha}\right)^{a} d t^{2}-d r^{2}-\left(\frac{r}{\alpha}\right)^{a} d \theta^{2}-\left(\frac{r}{\alpha}\right)^{2} d z^{2}+ \\
\quad+\frac{\epsilon t}{\alpha}\left(\left(\frac{r}{\alpha}\right)^{a} d t^{2}-d r^{2}-\left(\frac{r}{\alpha}\right)^{a} d \theta^{2}-\left(\frac{r}{\alpha}\right)^{2} d z^{2}\right), \quad a \neq 2 .
\end{aligned}
$$

Симметрии Нётер, соответствующие лагранжиану пространства-времени в уравнении (10), записываются как

$$
\begin{aligned}
\mathbf{X}_{1}= & \frac{\partial}{\partial s}, \quad \mathbf{X}_{2}=\frac{\partial}{\partial \theta}, \quad \mathbf{X}_{3}=\frac{\partial}{\partial z} \\
\mathbf{X}_{4}= & \frac{\partial}{\partial t}-\frac{\epsilon}{4 \alpha}\left((2-k) t \frac{\partial}{\partial t}+2 r \frac{\partial}{\partial r}+(2-k) \theta \frac{\partial}{\partial \theta}\right) \\
\mathbf{X}_{5}= & \frac{\partial}{\partial s}+\frac{r}{2} \frac{\partial}{\partial r}+\frac{2-a}{4} \theta \frac{\partial}{\partial \theta}+\frac{2-a}{4} z \frac{\partial}{\partial z}- \\
& \quad-\frac{\epsilon}{32 \alpha}\left(4 r^{2}\left(\frac{r}{\alpha}\right)^{-a}+t^{2}(2-a)^{2}+\theta^{2}(2-a)^{2} \frac{\partial}{\partial t}+\right. \\
& \left.+4 r t(2-a)^{2} \frac{\partial}{\partial r}+2 t \theta(2-k)^{2} \frac{\partial}{\partial \theta}\right) \\
\mathbf{X}_{6}= & \frac{\partial}{\partial t}+t \frac{\partial}{\partial \theta}-\frac{\epsilon}{4 \alpha}\left(t \theta(2-a) \frac{\partial}{\partial t}+2 r \theta \frac{\partial}{\partial r}+\right. \\
& \left.+\left(\frac{2 r^{2}}{a-2}\left(\frac{r}{\alpha}\right)^{-a}+\frac{t^{2}(2-a)}{2}+\frac{\theta^{2}(2-a)}{2}\right) \frac{\partial}{\partial \theta}\right) .
\end{aligned}
$$


ТАБЛИЦА 2

\begin{tabular}{|c|c|}
\hline Симметрии & Первые интегралы \\
\hline $\mathbf{X}_{4}$ & $\phi_{4}=2\left(\frac{r}{\alpha}\right)^{a} \dot{t}+\frac{\epsilon}{2 \alpha}\left(\left(\frac{r}{\alpha}\right)^{a} t \dot{t}(2-a)+2 r \dot{r}+\left(\frac{r}{\alpha}\right)^{a} \theta \dot{\theta}(2-a)\right)$ \\
\hline $\mathbf{X}_{5}$ & $\begin{aligned} \phi_{5}= & \left(\frac{r}{\alpha}\right)^{a} \frac{(2-a) t \dot{t}}{2}-r \dot{r}-\left(\frac{r}{\alpha}\right)^{a} \frac{(2-a) \theta \dot{\theta}}{2}+s \mathcal{L}- \\
& -\frac{\epsilon}{2 \alpha}\left(-t^{2} \dot{t}\left(\frac{r}{\alpha}\right)^{a} \frac{2-a}{2}+2 r t+t \theta \dot{\theta}\left(\frac{r}{\alpha}\right)^{a} \frac{2-a}{2}+\right. \\
& \left.+\frac{1}{16}\left(4 r^{2}\left(\frac{r}{\alpha}\right)^{-a}+t^{2}(2-a)^{2}+\theta^{2}(2-a)-4 r t(2-a)^{2}-4 t \theta(2-a)^{2}\right)\right)\end{aligned}$ \\
\hline $\mathbf{X}_{6}$ & $\begin{aligned} \phi_{6}=2\left(\frac{r}{\alpha}\right)^{a}(\theta \dot{t}-\dot{\theta} t)-\frac{\epsilon}{2 \alpha}(\theta \dot{t} t(2-a)-2 r \dot{r} \theta & -\left(\frac{r}{\alpha}\right)^{a} \dot{\theta}\left(\frac{2 r^{2}}{a-2}\left(\frac{r}{\alpha}\right)^{-a}+\right. \\
& \left.\left.+\frac{t^{2}(2-a)}{2}+\frac{\theta^{2}(2-a)}{2}\right)\right)\end{aligned}$ \\
\hline
\end{tabular}

ТАБЛИЦА 3

\begin{tabular}{|c|l|}
\hline Симметрии & Первые интегралы \\
\hline $\mathbf{X}_{4}$ & $L=2\left(1+\frac{\epsilon t}{\alpha}\right)\left(\frac{r}{\alpha}\right)^{a}(\theta \dot{z}-z \dot{\theta})$ \\
\hline $\mathbf{X}_{5}$ & $E=2\left(\left(\frac{r}{\alpha}\right)^{2} \dot{t}+\frac{\epsilon}{\alpha}\left(t \dot{t}\left(\frac{r}{\alpha}\right)^{2}+\frac{r \dot{r}}{2}+(2-a) \frac{\theta \dot{\theta}}{4}\left(\frac{r}{\alpha}\right)^{a}\right)+(2-a) \frac{z \dot{z}}{4}\left(\frac{r}{\alpha}\right)^{a}\right)$ \\
\hline $\mathbf{X}_{6}$ & Scaling $=-\left(\frac{r}{\alpha}\right)^{a} \frac{(2-a) z \dot{z}}{2}-r \dot{r}-\left(\frac{r}{\alpha}\right)^{a} \frac{(2-a) \theta \dot{\theta}}{2}+s \mathcal{L}$ \\
\hline
\end{tabular}

Законы сохранения или первые интегралы, соответствующие приближенным симметриям (11), приводятся в табл. 2.

2. Второй класс для шести симметрий Нётер:

$$
\begin{aligned}
d s^{2}=\left(\frac{r}{\alpha}\right)^{2} d t^{2}-d r^{2}-\left(\frac{r}{\alpha}\right)^{a} d \theta^{2}-\left(\frac{r}{\alpha}\right)^{a} d z^{2}+ \\
\quad+\frac{\epsilon t}{\alpha}\left(\left(\frac{r}{\alpha}\right)^{2} d t^{2}-d r^{2}-\left(\frac{r}{\alpha}\right)^{a} d \theta^{2}-\left(\frac{r}{\alpha}\right)^{a} d z^{2}\right), \quad a \neq 2 .
\end{aligned}
$$

Симметрии Нётер, соответствующие лагранжиану пространства-времени (12), имеют вид

$$
\begin{aligned}
& \mathbf{X}_{1}=\frac{\partial}{\partial s}, \quad \mathbf{X}_{2}=\frac{\partial}{\partial \theta}, \quad \mathbf{X}_{3}=\frac{\partial}{\partial z}, \quad \mathbf{X}_{4}=z \frac{\partial}{\partial \theta}-\theta \frac{\partial}{\partial z} \\
& \mathbf{X}_{5}=\frac{\partial}{\partial t}-\frac{\epsilon}{4 \alpha}\left(2 r \frac{\partial}{\partial r}+\theta(2-a) \frac{\partial}{\partial \theta}+z(2-a) \frac{\partial}{\partial z}\right) \\
& \mathbf{X}_{6}=s \frac{\partial}{\partial s}+\frac{r}{2} \frac{\partial}{\partial r}+\frac{2-a}{4} \theta \frac{\partial}{\partial \theta}+\frac{2-a}{4} z \frac{\partial}{\partial z} .
\end{aligned}
$$

Первые интегралы, соответствующие симметриям (13), приведены в табл. 3. 
ТАБЛИЦА 4

\begin{tabular}{|c|c|}
\hline Симметрии & Первые интегралы \\
\hline $\mathbf{X}_{4}$ & $L=\left(1+\frac{\epsilon t}{\alpha}\right)\left(\frac{r}{\alpha}\right)^{2}(\theta \dot{z}-z \dot{\theta})$ \\
\hline $\mathbf{X}_{5}$ & $E=\left(\frac{r}{\alpha}\right)^{a} \dot{t}+\frac{\epsilon}{\alpha}\left(\left(\frac{r}{\alpha}\right)^{a}\left(t \dot{t}-\frac{(2-a) t \dot{t}}{4}\right)+\frac{r \dot{r}}{2}\right)$ \\
\hline $\mathbf{X}_{6}$ & $\phi_{1}=2\left(\frac{r}{\alpha}\right)^{a}\left(\frac{(2-a) t \dot{t}}{4}-2 \dot{t}^{2} s\right)-r \dot{r}+2 s \dot{r}^{2}+s L+$ \\
& $+\frac{\epsilon}{\alpha}\left(-\frac{r^{2} \dot{t}}{4}+\left(\frac{r}{\alpha}\right)^{a}\left(-\frac{7 t^{2} \dot{t}(2-a)}{16}\right)+2\left(\frac{r}{\alpha}\right)^{a} t \dot{t}^{2} s-\frac{r \dot{r}(1+a)}{2}+2 t s \dot{r}^{2}\right)$ \\
\hline
\end{tabular}

3. Лагранжиан, который задает геодезические, следует из метрики пространства-времени

$$
\begin{aligned}
d s^{2}=\left(\frac{r}{\alpha}\right)^{a} d t^{2}-d r^{2}-\left(\frac{r}{\alpha}\right)^{2} d \theta^{2}-\left(\frac{r}{\alpha}\right)^{2} d z^{2}+ \\
\quad+\frac{\epsilon t}{\alpha}\left(\left(\frac{r}{\alpha}\right)^{a} d t^{2}-d r^{2}-\left(\frac{r}{\alpha}\right)^{2} d \theta^{2}-\left(\frac{r}{\alpha}\right)^{2} d z^{2}\right), \quad a \neq 2
\end{aligned}
$$

он допускает следующие симметрии Нётер:

$$
\begin{aligned}
\mathbf{X}_{1}= & \frac{\partial}{\partial s}, \quad \mathbf{X}_{2}=\frac{\partial}{\partial \theta}, \quad \mathbf{X}_{3}=\frac{\partial}{\partial z}, \quad \mathbf{X}_{4}=z \frac{\partial}{\partial \theta}-\theta \frac{\partial}{\partial z} \\
\mathbf{X}_{5}= & \frac{\partial}{\partial t}-\frac{\epsilon}{4 \alpha}\left((2-a) t \frac{\partial}{\partial t}+2 r \frac{\partial}{\partial r}\right) \\
\mathbf{X}_{6}= & s \frac{\partial}{\partial s}+\frac{2-a}{4} t \frac{\partial}{\partial t}+\frac{r}{2} \frac{\partial}{\partial r}- \\
& \quad-\frac{\epsilon}{32 \alpha}\left(\left(4 r^{2}\left(\frac{r}{\alpha}\right)^{-a}+t^{2}(a-2)^{2}\right) \frac{\partial}{\partial t}+4 r t(2-a) \frac{\partial}{\partial r}\right) .
\end{aligned}
$$

Соответствующие первые интегралы приведены в табл. 4.

4.2. Пространство-время с восемью симметриями Нётер и законы сохранения. Второй класс, допускающий восемь симметрий Нётер, имеет вид

$$
\begin{aligned}
d s^{2}=\left(\frac{r}{\alpha}\right)^{a} d t^{2}-d r^{2}-\left(\frac{r}{\alpha}\right)^{a} d \theta^{2}-\left(\frac{r}{\alpha}\right)^{a} d z^{2}+ & \\
& +\frac{\epsilon t}{\alpha}\left(\left(\frac{r}{\alpha}\right)^{a} d t^{2}-d r^{2}-\left(\frac{r}{\alpha}\right)^{a} d \theta^{2}-\left(\frac{r}{\alpha}\right)^{a} d z^{2}\right), \quad a \neq 2 .
\end{aligned}
$$


Соответствующие производящие операторы симметрий Нётер имеют вид

$$
\begin{aligned}
& \mathbf{X}_{1}=\frac{\partial}{\partial s}, \quad \mathbf{X}_{2}=\frac{\partial}{\partial \theta}, \quad \mathbf{X}_{3}=\frac{\partial}{\partial z}, \quad \mathbf{X}_{4}=z \frac{\partial}{\partial \theta}-\theta \frac{\partial}{\partial z} \\
& \mathbf{X}_{5}=\frac{\partial}{\partial t}-\frac{\epsilon}{8 \alpha}\left(2(2-a) t \frac{\partial}{\partial t}-2 x \frac{\partial}{\partial x}+2(2-a) y \frac{\partial}{\partial y}+2(2-a) z \frac{\partial}{\partial z}\right), \\
& \mathbf{X}_{6}=\theta \frac{\partial}{\partial t}+t \frac{\partial}{\partial \theta}-\frac{\epsilon}{8 \alpha}\left(2(2-a) t \theta \frac{\partial}{\partial t}\right)- \\
& -\frac{\epsilon}{8 \alpha}\left(4 r \theta \frac{\partial}{\partial r}-(a-2)\left(\frac{4 r^{2}}{(a-2)^{2}}\left(\frac{r}{\alpha}\right)^{-a}-\right.\right. \\
& \left.\left.-t^{2}+z^{2}-\theta^{2}\right) \frac{\partial}{\partial \theta}+2(2-a) \theta z \frac{\partial}{\partial z}\right) \\
& \mathbf{X}_{7}=z \frac{\partial}{\partial t}+t \frac{\partial}{\partial z}-\frac{\epsilon}{8 \alpha}\left(2(2-a) t z \frac{\partial}{\partial t}\right)- \\
& -\frac{\epsilon}{8 \alpha}\left(4 r z \frac{\partial}{\partial r}-(a-2)\left(\frac{4 r^{2}}{(a-2)^{2}}\left(\frac{r}{\alpha}\right)^{-a}-\right.\right. \\
& \left.\left.-t^{2}+\theta^{2}-z^{2}\right) \frac{\partial}{\partial z}+2(2-a) \theta z \frac{\partial}{\partial \theta}\right) \\
& \mathbf{X}_{8}=s \frac{\partial}{\partial s}+\frac{(2-a) t}{4} \frac{\partial}{\partial t}+\frac{r}{2} \frac{\partial}{\partial r}+\frac{(2-a) \theta}{4} \frac{\partial}{\partial \theta}+ \\
& +\frac{(2-a) z}{4} \frac{\partial}{\partial z}+\frac{\epsilon}{32 \alpha}\left(4 r^{2}\left(\frac{r}{\alpha}\right)^{-a}+(2-a)^{2}\left(t^{2}+\theta^{2}+z^{2}\right) \frac{\partial}{\partial t}-\right. \\
& \left.-4(a-2) \operatorname{tr} \frac{\partial}{\partial r}+2(a-2)^{2}\left(t \theta \frac{\partial}{\partial \theta}+t z \frac{\partial}{\partial z}\right)\right) \text {. }
\end{aligned}
$$

Оператор $\mathbf{X}_{5}$ соответствует энергии, $\mathbf{X}_{6}, \mathbf{X}_{7}$ соответствуют преобразованиям Лоренца (бусты), $\mathbf{X}_{8}$ соответствует скейлингу в данном пространстве-времени. Первые интегралы, соответствующие этим симметриям, даются в табл. 5.

4.2.1. Значения кривизны, соответствующие пространству-времени (16). Тензоры кривизны Римана, соответствующие пространству-времени (16), записываются как

$$
\begin{aligned}
& R_{0101}=\frac{(r / \alpha)^{a}\left(2 a^{2} t \epsilon+a^{2} \alpha-4 a t \epsilon-2 a \alpha\right)}{4 r^{2}(t \epsilon+\alpha)}, \\
& R_{0202}=\frac{(r / \alpha)^{2 a}\left(2 a^{2} t \epsilon+a^{2} \alpha\right)}{4 r^{2}(t \epsilon+\alpha)}=R_{0303}=-R_{2323}, \\
& R_{1313}=\frac{(r / \alpha)^{a}\left(2 a^{2} t \epsilon+a^{2} \alpha-4 a t \epsilon-2 a \alpha\right)}{4 r^{2}(t \epsilon+\alpha)}=R_{1212}, \\
& R_{0212}=-\frac{a \epsilon(r / \alpha)^{a}}{r \alpha}=R_{0313} .
\end{aligned}
$$

Из этих выражений ясно, что для $a<1$ пространство-время (16) является асимптотически плоским и подчиняется правилу, согласно которому волна затухает на значительном удалении от источника. 
ТАБЛИЦА 5

\begin{tabular}{|c|c|}
\hline Симметрии & Первые интегралы \\
\hline $\mathbf{X}_{4}$ & $L=\left(1+\frac{\epsilon t}{\alpha}\right)\left(\frac{r}{\alpha}\right)^{a}(\theta \dot{z}-z \dot{\theta})$ \\
\hline $\mathbf{X}_{5}$ & $\begin{aligned} E=\left(\frac{r}{\alpha}\right)^{a} \dot{t}+\frac{\epsilon}{\alpha}\left(\left(\frac{r}{\alpha}\right)^{a}\left(t \dot{t}-\frac{(2-a) t \dot{t}}{4}\right)+\right. & \\
& \left.+\frac{r \dot{r}}{2}+\left(\frac{r}{\alpha}\right)^{a}\left(\frac{(2-a) \theta \dot{\theta}}{4}+\frac{(2-a) z \dot{z}}{4}\right)\right)\end{aligned}$ \\
\hline $\mathbf{X}_{6}$ & $\begin{array}{r}\text { Boost }_{\theta}=2\left(\frac{r}{\alpha}\right)^{a}(\theta \dot{t}+\dot{\theta} t)+\frac{\epsilon}{\alpha}\left(\left(\frac{r}{\alpha}\right)^{a} \frac{(2+b) \theta t \dot{t}}{2}+\left(\frac{r}{\alpha}\right)^{a}\left(t^{2} \dot{\theta}-\dot{\theta}(a-2) \times\right.\right. \\
\left.\left.\quad \times\left(\frac{r^{2}}{(a-2)^{2}}\left(\frac{r}{\alpha}\right)^{-a}-t^{2}-\theta^{2}+z^{2}\right)+\frac{\theta z^{2} \dot{z}(a-2)}{4}\right)\right)\end{array}$ \\
\hline $\mathbf{X}_{7}$ & $\begin{aligned} \text { Boost }_{z}=2\left(\frac{r}{\alpha}\right)^{a}(z \dot{t} & +\dot{z} t)+\frac{\epsilon}{\alpha}\left(\left(\frac{r}{\alpha}\right)^{a} \frac{(2+b) z t \dot{t}}{2}+\left(\frac{r}{\alpha}\right)^{a}\left(t^{2} \dot{z}-\dot{z}(a-2) \times\right.\right. \\
& \left.\left.\times\left(\frac{r^{2}}{(a-2)^{2}}\left(\frac{r}{\alpha}\right)^{-a}-t^{2}-\theta^{2}+z^{2}\right)+\frac{\theta^{2} z \dot{\theta}(a-2)}{4}\right)\right)\end{aligned}$ \\
\hline $\mathbf{X}_{8}$ & $\begin{array}{r}\text { Scaling }=2\left(\frac{2-a}{2}\left(\left(\frac{r}{\alpha}\right)^{a} t \dot{t}-\left(\frac{r}{\alpha}\right)^{a} \theta \dot{\theta}-\left(\frac{r}{\alpha}\right)^{a} z \dot{z}\right)-2 s\left(\left(\frac{r}{\alpha}\right)^{a} \dot{t}^{2}-\right.\right. \\
\left.-\left(\frac{r}{\alpha}\right)^{a} \dot{\theta}^{2}-\left(\frac{r}{\alpha}\right)^{a} \dot{z}^{2}\right)-r \dot{r}+2 s \dot{r}^{2}+s \mathcal{L}+\frac{\epsilon}{\alpha}\left(\left(\frac{r}{\alpha}\right)^{a}\left(\frac{\dot{t}}{16}\left(4 r^{2}\left(\frac{r}{b}\right)^{-a}+\right.\right.\right. \\
\left.\left.+(2-a)^{2}\left(t^{2}+\theta^{2}+z^{2}\right)\right)+2 t \dot{t}\left(\frac{(2-a) t}{4}-s \dot{t}\right)\right)+\left(\frac{r}{\alpha}\right)^{a}\left(-\frac{\theta \dot{\theta} t(2-a)}{4}+\right. \\
\left.\left.\left.+2 t s \dot{\theta}^{2}-\frac{z \dot{z} t(2-a)}{4}+2 t s \dot{z}^{2}\right)+\frac{t r \dot{r}(a-6)}{4}+2 t s \dot{r}^{2}\right)\right)\end{array}$ \\
\hline
\end{tabular}

Соответствующие тензоры кривизны Риччи и скаляр Риччи даются выражениями

$$
\begin{aligned}
R_{00} & =-\frac{(r / \alpha)^{a}\left(6 a^{2} t \alpha \epsilon+3 a^{2} \alpha^{2}-4 a t \alpha \epsilon-2 a \alpha^{2}\right)}{4 r^{2}(t \epsilon+\alpha)}, \\
R_{12} & =-\frac{a \epsilon}{2 r(t \epsilon+\alpha)}, \quad R_{11}=\frac{3 a(a-2)}{4 r^{2}}, \quad R_{22}=\frac{a(3 a-2)(r / \alpha)^{a}}{4 r^{2}}=R_{33}, \\
R_{s} & =-\frac{3 a \alpha^{2}(2 a t \epsilon+a \alpha-2 t \epsilon-\alpha)}{r^{2}(t \epsilon+\alpha)^{3}} .
\end{aligned}
$$

Для $a<2$ пространство-время (16) является асимптотически риччи-плоским. Так как пространство-время (16) является нестатическим цилиндрически-симметричным пространством-временем, оно является цилиндрически-симметричным, приближенно риччи-плоским пространством-временем типа гравитационной волны.

4.2.2. Волновое уравнение в пространстве-времени (16). Волновое уравнение может быть записано как

$$
g^{\mu \nu} \nabla_{\mu} \nabla_{\nu} \Psi=0
$$


Оно упрощается и принимает вид

$$
\nabla_{\mu} \nabla^{\mu} \Psi=0
$$

С использованием частных производных имеем

$$
\frac{1}{\sqrt{-g}} \partial_{\mu}\left(\sqrt{-g} g^{\mu \nu} \partial_{\nu}\right) \Psi=0
$$

Подставляя

$$
\begin{aligned}
g_{00} & =\left(1+\frac{\epsilon t}{\alpha}\right)\left(\frac{r}{\alpha}\right)^{a}, & & g_{11}=-\left(1+\frac{\epsilon t}{\alpha}\right) \\
g_{22} & =-\left(1+\frac{\epsilon t}{\alpha}\right)\left(\frac{r}{\alpha}\right)^{a}, & g_{33} & =-\left(1+\frac{\epsilon t}{\alpha}\right)\left(\frac{r}{\alpha}\right)^{a}, \\
g^{00} & =\left(1-\frac{\epsilon t}{\alpha}\right)\left(\frac{r}{\alpha}\right)^{-a}, & g^{11} & =-\left(1-\frac{\epsilon t}{\alpha}\right), \\
g^{22} & =-\left(1-\frac{\epsilon t}{\alpha}\right)\left(\frac{r}{\alpha}\right)^{-a}, & g^{33} & =-\left(1-\frac{\epsilon t}{\alpha}\right)\left(\frac{r}{\alpha}\right)^{-a}
\end{aligned}
$$

в (22), мы получаем волновое уравнение в пространстве-времени (16):

$$
\begin{aligned}
& \left(\left(\frac{r}{\alpha}\right)^{a}\left(\partial_{t t}-\partial_{\theta \theta}-\partial_{z z}\right)-\partial_{r r}-\frac{3 a}{2}\left(\frac{\alpha}{r}\right) \partial_{r}-\right. \\
& \left.\quad-\frac{\epsilon}{\alpha}\left(\left(\frac{r}{\alpha}\right)^{a}\left(t \partial_{t t}-t \partial_{\theta \theta}-t \partial_{z z}-\partial_{t}\right)-t \partial_{r r}-\frac{3 a t}{2}\left(\frac{\alpha}{r}\right) \partial_{r}\right)\right) \Psi=0 .
\end{aligned}
$$

4.3. Девять симметрий Нётер. Лагранжиан метрики

$$
\begin{aligned}
d s^{2}=\left(\frac{r}{\alpha}\right)^{2} d t^{a}-d r^{2}-\left(\frac{r}{\alpha}\right)^{2} d \theta^{2}-\left(\frac{r}{\alpha}\right)^{2} d z^{2}+ \\
+\frac{\epsilon t}{\alpha}\left(\left(\frac{r}{\alpha}\right)^{2} d t^{2}-d r^{2}-\left(\frac{r}{\alpha}\right)^{2} d \theta^{2}-\left(\frac{r}{\alpha}\right)^{2} d z^{2}\right)
\end{aligned}
$$

дает девять симметрий Нётер, четыре из которых имеют приближенные части. Точные симметрии даются выражениями

$$
\begin{aligned}
& \mathbf{X}_{1}=\frac{\partial}{\partial s}, \quad \mathbf{X}_{2}=\frac{\partial}{\partial \theta}, \quad \mathbf{X}_{3}=\frac{\partial}{\partial z} \\
& \mathbf{X}_{4}=\theta \frac{\partial}{\partial z}-z \frac{\partial}{\partial \theta}, \quad \mathbf{X}_{5}=s \frac{\partial}{\partial s}+\frac{r}{2} \frac{\partial}{\partial r} \\
& \mathbf{X}_{6}=\frac{\partial}{\partial t}-\frac{\epsilon}{2 \alpha} r \frac{\partial}{\partial r}, \quad \mathbf{X}_{7}=s^{2} \frac{\partial}{\partial s}+r s \frac{\partial}{\partial r}-\frac{\epsilon \alpha}{2} s \frac{\partial}{\partial t} \\
& \mathbf{X}_{8}=\theta \frac{\partial}{\partial t}+t \frac{\partial}{\partial \theta}-\frac{\epsilon}{2 \alpha}\left(r \theta \frac{\partial}{\partial r}-\alpha^{2} \ln \left(\frac{r}{\alpha}\right) \frac{\partial}{\partial \theta}\right) \\
& \mathbf{X}_{9}=z \frac{\partial}{\partial t}+t \frac{\partial}{\partial z}-\frac{\epsilon}{2 \alpha}\left(r z \frac{\partial}{\partial r}-\alpha^{2} \ln \left(\frac{r}{\alpha}\right) \frac{\partial}{\partial z}\right)
\end{aligned}
$$

Первые интегралы, соответствующие этим симметриям Нётер, приводятся в табл. 6 . 
ТАБЛИЦА 6

\begin{tabular}{|c|l|}
\hline Симметрии & \multicolumn{1}{|c|}{ Первые интегралы } \\
\hline $\mathbf{X}_{4}$ & $L=\left(1+\frac{\epsilon t}{\alpha}\right)\left(\frac{r}{\alpha}\right)^{2}(\theta \dot{z}-z \dot{\theta})$ \\
\hline $\mathbf{X}_{5}$ & Scaling $=-\left(1+\frac{\epsilon t}{\alpha}\right) r \dot{r}-s \mathcal{L}$ \\
\hline $\mathbf{X}_{6}$ & $\phi_{0}=2\left(\left(\frac{r}{\alpha}\right)^{2} \dot{t}+\frac{\epsilon}{\alpha} \frac{r \dot{r}}{2}\right)$ \\
\hline $\mathbf{X}_{7}$ & $\phi_{2}=-2\left(\frac{r}{\alpha}\right)^{2} s \dot{t}-2 r \dot{r} s+2 s^{2} \dot{r}^{2}+r^{2}-$ \\
\hline $\mathbf{X}_{8}$ & $\left.\phi_{4}=2\left(\frac{r}{\alpha}\right)^{2}(\theta \dot{t}-\dot{\theta} t)+\frac{\epsilon}{\alpha}\left(2\left(\frac{r}{\alpha}\right)^{2} t \dot{t} \theta-\left(\frac{r}{\alpha}\right)^{2} \dot{\theta} \alpha^{2} \ln \frac{r}{\alpha}-2 s t \dot{t}^{2}\right)+2 t \dot{r}\left(r s-s^{2} r\right)^{2}+r^{2} t\right)$ \\
\hline $\mathbf{X}_{9}$ & $\left.\phi_{5}=2\left(\frac{r}{\alpha}\right)^{2}(z \dot{t}-\dot{z} t)+\frac{\epsilon}{\alpha}\left(2\left(\frac{r}{\alpha}\right)^{2} t \dot{\theta} z-\left(\frac{r}{\alpha}\right)^{2} \dot{z}\right)^{2}+r \dot{r} \theta\right)$ \\
\hline
\end{tabular}

\section{5. ЗАКЛЮЧЕНИЕ}

После получения полного перечня возможных цилиндрически-симметричных пространств-времен (с помощью зависящего от времени приближенного фактора), где приближенные симметрии Нётер существуют для данного зависящего от времени конформного возмущения $e^{\epsilon f(t)}$, можно сделать следующие выводы.

1. Приближенная часть появляется в тех классах цилиндрически-симметричных пространств-времен, для которых действия плотностей лагранжианов допускают четыре, шесть, восемь или девять симметрий Нётер и, следовательно, четыре, шесть, восемь или девять законов сохранения.

2. Только временна́я трансляция, скейлинг и симметрия, соответствующие преобразованиям Лоренца, допускают приближенные части.

3. Действие лагранжианов пространств-времен с нулевой секционной кривизной не дает приближенных симметрий Нётер.

4. Для данного возмущения приближенная симметрия Нётер не существует в плоских пространствах-временах (например, в пространстве-времени Минковского).

Хорошо известно, что $K V \subseteq H V \subset N S$, где $K V$ - векторы Киллинга, $H V$ векторы гомотетии, $N S$ - симметрии Нётер. Такое соотношение для приближенных симметрий не встречалось в литературе. Однако на основе наших результатов 
можно выдвинуть гипотезу, что подобный результат будет выполняться для приближенных симметрий. Интересно классифицировать пространства-времена по их приближенным векторам Киллинга $(K V)$ и векторам гомотетии $(H V)$ для доказательства гипотезы.

В п. 4.2.1 и в соотношениях (20) показано, что тензор кривизны Римана $R_{\mu \nu \alpha \beta} \rightarrow 0$ при $r \rightarrow \infty$ для $a<1$, это демонстрирует, что гравитационная волна затухает асимптотически по мере того, как она удаляется от источника на большое расстояние. Это поведение пространства-времени (16) показывает, что оно прекрасно соответствует пространству-времени типа гравитационной волны. Первые интегралы даны в табл. 1-6, где коэффициенты при $\epsilon$ являются поправками к законам сохранения в соответствующих пространствах-временах.

\section{ПРИЛОЖЕНИЕ}

Система симметрий Нётер, определяющих дифференциальные уравнения в частных производных, имеет вид

$$
\begin{aligned}
& \xi_{\mathrm{a}, t}=0, \quad \xi_{\mathrm{a}, r}=0, \quad \xi_{\mathrm{a}, \theta}=0, \quad \xi_{\mathrm{a}, z}=0, \quad A_{\mathrm{a}, s}=0, \\
& f_{t}(t) \eta_{e}^{0}+\left(\eta_{a}^{1}+f(t) \eta_{e}^{1}\right) \nu(r)_{r}+2\left(\eta_{\mathrm{a}, t}^{0}+f(t) \eta_{\mathrm{e}, t}^{0}\right)-f(t) \xi_{\mathrm{e}, s}-\xi_{\mathrm{a}, s}=0, \\
& f_{t}(t) \eta_{e}^{0}+\left(\eta_{a}^{1}+f(t) \eta_{e}^{1}\right) \mu(r)_{r}+2\left(\eta_{\mathrm{a}, t}^{2}+f(t) \eta_{\mathrm{e}, t}^{2}\right)-f(t) \xi_{\mathrm{e}, s}-\xi_{\mathrm{a}, s}=0, \\
& f_{t}(t) \eta_{e}^{0}+\left(\eta_{a}^{1}+f(t) \eta_{e}^{1}\right) \lambda(r)_{r}+2\left(\eta_{\mathrm{a}, t}^{3}+f(t) \eta_{\mathrm{e}, t}^{3}\right)-f(t) \xi_{\mathrm{e}, s}-\xi_{\mathrm{a}, s}=0, \\
& f_{t}(t) \eta_{e}^{0}+\left(\eta_{\mathrm{a}, r}^{1}+f(t) \eta_{\mathrm{e}, r}^{1}\right)-f(t) \xi_{\mathrm{e}, s}-\xi_{\mathrm{a}, s}=0, \\
& \left(f(t) \eta_{\mathrm{e}, \theta}^{0}+\eta_{\mathrm{a}, \theta}^{0}\right) e^{\nu(r)}-\left(f(t) \eta_{\mathrm{e}, t}^{2}+\eta_{\mathrm{a}, t}^{2}\right) e^{\mu(r)}=0, \\
& \left(f(t) \eta_{\mathrm{e}, r}^{0}+\eta_{\mathrm{a}, r}^{0}\right) e^{\nu(r)}-\left(f(t) \eta_{\mathrm{e}, t}^{1}+\eta_{\mathrm{a}, t}^{1}\right)=0, \\
& \left(f(t) \eta_{\mathrm{e}, z}^{0}+\eta_{\mathrm{a}, z}^{0}\right) e^{\nu(r)}-\left(f(t) \eta_{\mathrm{e}, t}^{3}+\eta_{\mathrm{a}, t}^{3}\right) e^{\lambda(r)}=0, \\
& \left(f(t) \eta_{\mathrm{e}, r}^{2}+\eta_{\mathrm{a}, r}^{2}\right) e^{\mu(r)}+\left(f(t) \eta_{\mathrm{e}, \theta}^{1}+\eta_{\mathrm{a}, \theta}^{1}\right)=0, \\
& \left(f(t) \eta_{\mathrm{e}, z}^{2}+\eta_{\mathrm{a}, z}^{2}\right) e^{\mu(r)}-\left(f(t) \eta_{\mathrm{e}, \theta}^{3}+\eta_{\mathrm{a}, \theta}^{3}\right) e^{\lambda(r)}=0, \\
& \left(f(t) \eta_{\mathrm{e}, r}^{3}+\eta_{\mathrm{a}, r}^{3}\right) e^{\lambda(r)}+\left(f(t) \eta_{\mathrm{e}, z}^{1}+\eta_{\mathrm{a}, z}^{1}\right)=0, \\
& \left(f(t) \eta_{\mathrm{e}, s}^{0}+2 \eta_{\mathrm{a}, s}^{0}\right) e^{\nu(r)}-A_{\mathrm{a}, t}=0, \quad\left(f(t) \eta_{\mathrm{e}, s}^{1}+2 \eta_{\mathrm{a}, s}^{1}\right)+A_{\mathrm{a}, r}=0, \\
& \left(f(t) \eta_{\mathrm{e}, s}^{2}+2 \eta_{\mathrm{a}, s}^{2}\right) e^{\mu(r)}+A_{\mathrm{a}, \theta}=0, \quad\left(f(t) \eta_{\mathrm{e}, s}^{3}+2 \eta_{\mathrm{a}, s}^{3}\right) e^{\lambda(r)}+A_{\mathrm{a}, z}=0 .
\end{aligned}
$$

Закон сохранения, соответствующий каждой симметрии Нётер, определяется как

$$
\phi=\frac{\partial \mathcal{L}}{\partial \dot{x}^{i}}\left(\eta^{i}\left(s, x^{i}\right)-\xi \dot{x}^{i}\right)+\mathcal{L} D(\xi)=A
$$

где $D$ - полный дифференциальный оператор, $A$ - калибровочная функция, определенная в разделе 3 .

\section{Список литературы}

[1] W. H. Press, K.S. Thorne, "Gravitational-wave astronomy", Ann. Rev. Astron. Astrophys., 10 (1972), 335-374; У. Пресс, К. Торн, "Гравитационно-волновая астрономия", УФН, 110:569-606 (1973).

[2] R.H. Price, K.S. Thorne, "Nonradial pulsation of general relativistic stellar models. II. Properties of the gravitational waves", Astrophys. J., 155 (1969), 163-182. 
[3] K. S. Thorne, "Gravitational radiation damping", Phys. Rev. Lett., 21:5 (1968), 320-323.

[4] K. S. Thorne, "Sources of gravitational waves", Proc. Roy. Soc. London A, 368:1732 (1979), $9-10$.

[5] K. S. Thorne, "Gravitational-wave research: current status and future prospects", Rev. Modern Phys., 52:2 (1980), 285-298.

[6] E. L. Hill, "Hamilton's principle and the conservation theorems of mathematical physics", Rev. Modern Phys., 23:3 (1951), 253-260.

[7] E. Noether, "Invariante Variationsprobleme", Nachr. Ges. Wiss. Göttingen, Math.-Phys. Kl., 2 (1918), 235-275.

[8] A. Trautman, "Conservation laws in general relativity", Gravitation: An Introduction to Current Research, ed. L. Witten, John Wiley and Sons, New York, 1962, 169-198.

[9] A. Einstein, N. Rosen, "On gravitational waves", J. Franklin Inst., 223:1 (1937), 43-54.

[10] F. Ali, T. Feroze, "Classification of plane symmetric static space-times according to their Noether's symmetries", Internat. J. Theor. Phys., 52:9 (2013), 3329-3342.

[11] Ф. Али, Т. Ферозе, С. Али, "Полная классификация сферически-симметричных статических пространств-времен с помощью симметрий Нётер”, ТМФ, 184:1 (2015), 92-105.

[12] П. Олвер, Приложение групп Ли к дифференциальным уравнениям, Мир, М., 1989.

[13] F. Ali, "Conservation laws of cylindrically symmetric vacuum solution of Einstein field equations", Appl. Math. Sci., 8:94 (2014), 4697-4702.

[14] U. Camci, A. Yildirim, "Lie and Noether symmetries in some classes of pp-wave spacetime", Phys. Scr., 89 (2014), 084003, 8 pp.

[15] U. Camci, "Symmetries of geodesic motion in Gödel-type spacetimes", JCAP, 2014:07 (2014), 002, 20 pp.

[16] Y. Kucukakca, U. Camci, "Noether gauge symmetry for $f(R)$ gravity in Palatini formalism", Astrophys. Space Sci., 338:1 (2012), 211-216.

[17] S. Capozziello, R. de Ritis, P. Scudellaro, "Nöther symmetries in quantum cosmology", Internat. J. Modern Phys. D, 3:3 (1994), 609-621.

[18] S. Capozziello, M. De Laurentis, S. D. Odintsov, "Hamiltonian dynamics and Noether symmetries in extended gravity cosmology", Eur. Phys. J. C, 72 (2012), 2068, 21 pp.

[19] F. Ali, T. Feroze, "Approximate Noether symmetries from geodetic Lagrangian for plane symmetric spacetimes", Internat. J. Geom. Modern Phys., 12:1550124 (2015), 9 pp.

[20] N. H. Ibragimov, Elementary Lie Group Analysis and Ordinary Differential Equations, Wiley Series in Mathematical Methods in Practice, 4, John Wiley and Sons, Chichester, 1999.

[21] G. Ünal, "Approximate generalized symmetries, normal forms and approximate first integrals", Phys. Lett. A, 269:1 (2000), 13-30.

[22] G. Ünal, C. M. Khalique, G.F. Alişveriş̧̧i, "Approximate first integrals of a chaotic Hamiltonian system", Quaest. Math., 30:4 (2009), 483-497.

[23] G. Ünal, C. M. Khalique, "Approximate conserved quantities of conservative dynamical systems in $R^{3 ",}$ Quaest. Math., 28:3 (2005), 305-315.

[24] T. Levi-Civita, "IX: L'analogo del potenziale logaritmico", Rom. Acc. L. Rend. (5), 28:1 (1919), 101-109.

[25] В. Д. Захаров, Гравитационные волны в теории тяготения Эйнштейна, Наука, М., 1972.

[26] A. Jawad, F. Ali, S. M. Umair, G. Abbas, "Dynamics of particles around time conformal Schwarzschild black hole", Eur. Phys. J. C, 76 (2016), 586, 12 pp.

[27] D. Kramer, H. Stephani, E. Herlt, M. A. H. MacCallum, Exact Solution of Einstein's Field Equations, Cambridge Monographs on Mathematical Physics, 6, Cambridge Univ. Press, Cambridge, 1980.

[28] F. Ali, T. Feroze, "Complete classification of cylindrically symmetric static spacetime and the corresponding conservation laws", Mathematics, 4:3 (2016), 50, 15 pp. 